\title{
Kegler, Karl R. (2015): Deutsche Raumplanung. Das Modell der „Zentralen Orte“ zwischen NS-Staat und Bundesrepublik
}

\author{
Paderborn: Verlag Ferdinand Schöningh. 645 Seiten
}

\section{Oliver Werner ${ }^{1}$ iD}

Online publiziert: 15 . August 2016

(c) Springer-Verlag Berlin Heidelberg 2016

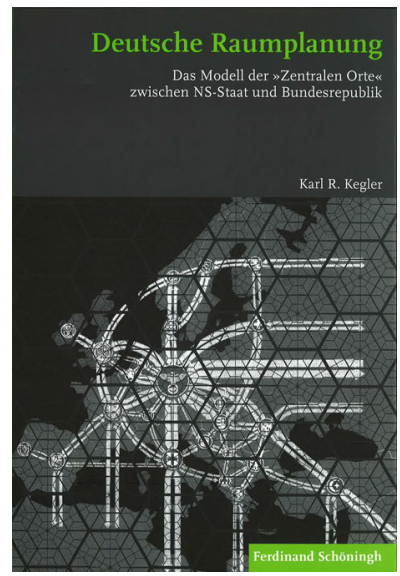

Das Verhältnis zwischen Zeitgeschichte und Raumwissenschaft ist - gelinde gesagt - schwierig. Während sich Zeithistoriker seit den 1990er-Jahren intensiv mit der Entwicklung der deutschen Raumforschung und Landesplanung im Nationalsozialismus und in der frühen Bundesrepublik befassen (etwa Aly/Heim 1991; Heinemann 2003; Leendertz 2008), haben sich Raumwissenschaftler lange mit diesem Themenkomplex schwer getan. Zwar legten sie sporadisch wichtige Studien vor (Gröning/Wolschke-Bulmahn 1987; Rössler/Schleiermacher 1993), indes entstanden erst in jüngerer Zeit - zumeist unter der Federführung der Akademie für Raumforschung und Landesplanung (ARL) - Arbeiten, die thematisch breit und interdisziplinär die Rolle der Raumwissenschaften im „Dritten Reich“ untersuchen

Dr. Oliver Werner

werner@idd.uni-hannover.de

1 Institut für Didaktik der Demokratie, Leibniz Universität Hannover, Schlosswender Straße 1, 30159 Hannover, Deutschland und das Ausmaß personeller und inhaltlicher Kontinuitäten von den Umgestaltungsplanungen für ein besetztes Europa in die Bundesrepublik Deutschland bestimmen (Mäding/ Strubelt 2009; Strubelt/Briesen 2015).

Eine grundsätzliche Auseinandersetzung der Raumwissenschaften mit der eigenen Geschichte war sehr lange nur mühsam in Gang zu bringen, nicht zuletzt auch, wie ein Präsident der ARL in den 1980er-Jahren meinte, weil „noch zu viele aus der damaligen Zeit lebten“ (Strubelt/Briesen 2015: 10). Mit der Dissertation des Architekten und Stadthistorikers Karl R. Kegler liegt nun für die Weiterführung dieser wichtigen Debatte eine solide und sehr anregende Grundlage vor.

Kegler untersucht am Beispiel der Rezeption von Walter Christallers Modell der „Zentralen Orte“ die Geschichte der deutschen Raumplanung zwischen 1930 und 1970. Im Mittelpunkt steht die Frage, wie es zu erklären sei, „dass Christallers Modell trotz der benennbaren schwerwiegenden theoretischen Mängel in ganz unterschiedlichen Gesellschaftssystemen zu einem weithin akzeptierten theoretischen und normativen Instrument der Wissenschaft und Planungspraxis wurde" (S. 11).

Das Buch teilt sich in drei große Hauptkapitel. Im ersten Teil „Die geplante Ordnung“ arbeitet Kegler die Widersprüche und Defizite des Zentrale-Orte-Modells von Walter Christaller heraus, dem er einen plausiblen theoretischen Gehalt abspricht. Tatsächlich wird erkennbar, dass Christallers Ansatz von dem fundamentalen Widerspruch geprägt ist, dass es als ein Modell, ,das ursprünglich als reines Erklärungsmodell für die siedlungsgeographische Wirklichkeit konzipiert war“, durch „raumordnungspolitische Entwicklungsziele [...] zum Leitbild der Raumentwicklung“ (S. 69) erhoben wurde. Damit ist zwar der Stab über dem Zentrale-Orte-Modell gebrochen, aber die wissenschaftliche und politische Attraktivität von Christallers 
Ansatz noch nicht erklärt. Diese habe vielmehr auf der zeitgenössischen „Kompatibilität der Zentrale-Orte-Theorie mit dem geographischen Landschaftsparadigma und national-konservativen Anschauungen der deutschen Geographie“ (S. 110 f.) beruht. In der Folge sei dann gerade die aus „Denkfehlern und Widersprüchen“ hervorgehende „Mehrdeutigkeit ein Element für den fachübergreifenden Erfolg des Modells geworden“ (S. 486).

Im zweiten Hauptkapitel „Geometrie für den totalen Staat“" schildert Kegler die Rezeption des Zentrale-OrteModells im Kontext der sich entfaltenden Raumwissenschaften im nationalsozialistischen Deutschland. Sowohl die 1935 gebildete „Reichsarbeitsgemeinschaft für Raumforschung" als auch der Planungsapparat Heinrich Himmlers in dessen Funktion als „Reichskommissar für die Festigung des deutschen Volkstums" (RKF) erwiesen sich als einflussreiche Rezipienten des Zentrale-Orte-Modells, sodass Kegler eine ,grundlegende Akzeptanz des Modells als Grundlage für die Neugliederung und bleibende Inbesitznahme der besetzten Gebiete in Osteuropa“ (S. 156) feststellt. Allerdings lassen sich die Bezüge zwischen Christallers Überlegungen und insbesondere den verschiedenen Varianten des „Generalplans Ost“ aufgrund der Quellenlage oft nur indirekt bestimmen. Demgegenüber ist Christallers persönlicher Einsatz für sein Modell als Mitarbeiter beim RKF sehr gut nachweisbar, und sein Vorgehen belegt deutlich ,den willkürlichen Gebrauch und die forcierte Überspannung der Begründungspotentiale“ (S. 192) seiner Überlegungen.

Das dritte Hauptkapitel „Von Ordnung zum Ausgleich“ untersucht schließlich die Rezeption des Zentrale-OrteModells in der Bundesrepublik Deutschland bis zum Ende der 1960er-Jahre. Dabei wird eine Konjunktur des Begriffes „Zentrale Orte“ konterkariert von der wachsenden Distanzierung der Raumwissenschaften von Christallers Methodik, und gerade die ,variantenreiche Suche nach der empirischen Verifizierbarkeit des Christallerschen Modells“ verdeutliche ,einen entscheidenden Unterschied zu seiner Anwendung in der nationalsozialistischen Raumplanung" (S. 356). Damit steht die wesentliche, von Kegler keineswegs abschließend beantwortete Frage im Raum, was Christallers „Zentrale Orte“ vom Beginn der 1930er-Jah- re noch mit den westdeutschen Raumordnungsplänen der 1960er-Jahre gemeinsam haben. Zum einen belegt Kegler, dass Christallers Modellvorstellungen von vielen Raumwissenschaftlern, etwa des „Instituts für Raumforschung“, abgelehnt wurden, zum anderen häufen sich Beispiele, in denen die ,zentralen Orte“ ein begriffliches Eigenleben entwickeln, ohne sich überhaupt noch namentlich auf Walter Christaller zu beziehen (Wensel 1963).

Die Bedeutung des Zentrale-Orte-Modells beruhte in erster Linie nicht auf inspirierenden Methoden oder einer theoretischen Schlüssigkeit, sondern vielmehr, wie Kegler umfassend herausarbeitet, auf einer Adaptions- und Anschlussfähigkeit an ganz verschiedene politische Absichten und Herrschaftskonzeptionen und auf einer enormen begrifflichen Suggestivkraft. Walter Christaller hatte wohl vor allem ein Gespür für einprägsame Begriffe, während seine theoretischen Überlegungen über die Jahre und Jahrzehnte verblichen sind.

\section{Literatur}

Aly, G.; Heim, S. (1991): Vordenker der Vernichtung. Auschwitz und die deutschen Pläne für eine neue europäische Ordnung. Hamburg.

Gröning, G.; Wolschke-Bulmahn, J. (1987): Der Drang nach Osten. Zur Entwicklung der Landespflege im Nationalsozialismus und während des 2. Weltkrieges in den ,eingegliederten Ostgebieten“. München.

Heinemann, I. (2003): „Rasse, Siedlung, deutsches Blut“. Das Rasseund Siedlungshauptamt der SS und die rassenpolitische Neuordnung Europas. Göttingen.

Leendertz, A. (2008): Ordnung schaffen. Deutsche Raumplanung im 20. Jahrhundert. Göttingen.

Mäding, H.; Strubelt, W. (Hrsg.) (2009): Vom Dritten Reich zur Bundesrepublik. Beiträge einer Tagung zur Geschichte von Raumforschung und Raumplanung. Hannover. = Arbeitsmaterial der ARL 346.

Rössler, M.; Schleiermacher, S. (Hrsg.) (1993): Der „Generalplan Ost“. Hauptlinien der nationalsozialistischen Planungs- und Vernichtungspolitik. Berlin.

Strubelt, W.; Briesen, D. (Hrsg.) (2015): Raumplanung nach 1945. Kontinuitäten und Neuanfänge in der Bundesrepublik Deutschland. Frankfurt am Main, New York.

Wensel, W. (1963): Die Zentralen Orte in Niedersachsen. Beitrag zur Frage einer Einordnung der Zentralen Orte in Rangstufen. In: Neues Archiv für Niedersachsen 12, 190-204. 